\title{
El fluoruro en aguas de consumo y su asociación con variables geológicas y geográficas de Cuba
}

\author{
Liliam Cuéllar Luna ${ }^{1}$ y Maricel García Melián ${ }^{1}$
}

RESUMEN Objetivo. Determinar la asociación entre las diferentes concentraciones del ión fluoruro en aguas de consumo y algunas variables geológicas y geográficas de Cuba, mediante el empleo de un sistema de información geográfica.

Métodos. De noviembre de 1998 a octubre de 1999 se estudiaron las concentraciones de fluoruro en fuentes de abastecimiento de agua de consumo de 753 localidades cubanas de 1000 habitantes o más. Para el análisis de la información se utilizó el sistema de información geográfica MapInfo Professional (v. 5.5) con el método de superposición. Las variables de estudio fueron la concentración del ión fluoruro en fuentes de abastecimiento de agua, las características geológicas del territorio, los alineamientos, los tipos de fuente y las categorías de relieve (llanura o montaña). Los resultados se agruparon por localidad y municipio.

Resultados. En $83,1 \%$ de las localidades, las muestras de agua fueron recolectadas en pozos y manantiales y en el 16,9\% restante, en presas y ríos. De las 753 localidades estudiadas, 675 $(89,6 \%)$ presentaron concentraciones de fluoruro bajas o medias $(<0,7 \mathrm{mg} / \mathrm{L})$. La región oriental del país fue la más afectada por concentraciones elevadas de fluoruro en las aguas, seguida de la región central. La mayoría de las localidades con altas concentraciones de fluoruro natural se localizaron en zonas ubicadas sobre rocas del arco volcánico del Cretácico. La presencia de fluoruro en las aguas de consumo estuvo asociada con los alineamientos de la corteza terrestre, no solo en los complejos rocosos de origen volcánico-sedimentario e intrusivo, sino también en las rocas carbonatadas, aunque se debe destacar que las mayores concentraciones de fluoruro coincidieron fundamentalmente con los dos primeros complejos rocosos mencionados anteriormente. Todas las localidades con concentraciones altas de fluoruro en el agua estaban vinculadas a pozos.

Conclusiones. La concentración de fluoruro en las fuentes de agua de consumo de 89,6\% de las poblaciones cubanas con 1000 habitantes o más es baja o mediana. Las características geológicas y geográficas pueden ayudar a identificar zonas con concentraciones óptimas y altas del ión fluoruro en las aguas de consumo.

Palabras clave Caries dental, fluoruración, geología, Cuba.

Instituto Nacional de Higiene, Epidemiología y Microbiología, Ciudad de La Habana, Cuba. La correspondencia debe enviarse a: Liliam Cuéllar Luna, Instituto Nacional de Higiene, Epidemiología y Microbiología, Calzada de Infanta No. 1158 entre Llinás y Clavel, Centro Habana, Ciudad de La Habana, Cuba. Correo electrónico: lcuellar@inhem. sld.cu.
La caries dental es una enfermedad que afecta a la salud de millones de personas en el mundo. En la Región de las Américas, la proporción de personas que han sido afectadas por la caries dental en la década de 1990 es muy elevada; entre los escolares de 5 a
17 años, la proporción es de 90\% aproximadamente (1).

Se sabe que el consumo controlado de fluoruro ayuda a disminuir la caries dental $(2,3)$, lo que ha permitido reducir esta afección en los países donde se agrega fluoruro al agua o a la sal. En 
la actualidad, estos programas forman parte de las estrategias de salud pública y contribuyen considerablemente a la reducción de la caries dental a un costo bajo (1). En Cuba se estableció el Programa de Fluoración de la Sal de Consumo, a cargo de la Dirección Nacional de Estomatología del Ministerio de Salud Pública. La Organización Panamericana de la Salud (OPS) apoyó este programa mediante un proyecto de estudio de factibilidad realizado en 1996.

Para poner en marcha este programa era necesario determinar las fuentes naturales de fluoruro y localizar los territorios con concentraciones óptimas y altas de este ión en sus aguas de consumo. En esas localidades no se debe distribuir sal fluorurada para evitar efectos adversos a la salud debido al consumo excesivo de fluoruro (desde una fluorosis dental leve hasta una fluorosis esquelética discapacitante).

Los autores del estudio del contenido de fluoruro en aguas de consumo realizado en Cuba en la década de 1950 concluyeron que las concentraciones de estas sales eran generalmente bajas y que las concentraciones altas se localizaban solo en algunas zonas del país (4).

En 1973 en Cuba se decidió comenzar a fluorurar el agua de consumo, y la primera instalación se estableció en La Salud, comunidad de la provincia de La Habana. En 1975 se acordó llevar a cabo, en colaboración con el Fondo de las Naciones Unidas para la Infancia (UNICEF) y la Organización Mundial de la Salud (OMS), un programa limitado de fluoruración del agua de consumo mediante el cual se beneficiaron 9 comunidades: 7 de La Habana y 2 de Ciudad de La Habana. Posteriormente se implementó este tratamiento en los acueductos de San Luis, en la provincia de Pinar del Río, y Viñas, en Villa Clara (5).

Como resultado de este programa limitado, en la provincia La Habana se apreció la reducción del índice de caries (COP) en tres comunidades donde se consumía agua fluorurada desde hacía más de 5 años y se llegó a la conclusión de que el programa había logrado reducir la incidencia de caries dental en la población infantil estu- diada (5). A pesar del impacto positivo en la población que fue objeto de la intervención, no se continuó la aplicación de esta técnica por falta de recursos.

En 1998 se retomaron los estudios mediante un proyecto de colaboración entre la Dirección Nacional de Estomatología del Ministerio de Salud Pública y el Instituto Nacional de Higiene, Epidemiología y Microbiología, cuyo propósito fue determinar las concentraciones del ión fluoruro en las aguas de consumo. Para ello se muestrearon todas las fuentes de abastecimiento de agua en las localidades de 1000 o más habitantes, las cuales posteriormente se clasificaron de acuerdo con las concentraciones de fluoruro, según las normas establecidas por la OMS: bajas (de 0 a 0,39 mg/L), medias (de 0,4 a 0,69 mg/L), óptimas (de 0,7 a 1,49 mg/L) y altas (1,50 mg/L o más).

El flúor puede llegar al agua con el polvo, el carbón incinerado, las emanaciones volcánicas, etc. Sin embargo, la proporción de fluoruro que llega a los recursos hídricos por esta vía es extremadamente pequeña en comparación con la procedente de la lixiviación de las rocas y los suelos. De ahí que las concentraciones de fluoruro en las aguas de consumo estén en estrecha relación con las características geológicas de cada territorio $(2,3,6)$.

Para analizar la constitución geológica de Cuba es importante saber que la configuración geográfica actual del archipiélago no fue siempre la misma y que en las diferentes etapas de su desarrollo algunas rocas que componen la geología actual no estaban formadas aún, se estaban depositando o formaban parte de otras estructuras del planeta. Esta constitución geológica se puede representar mediante un modelo en el que se reconocen dos niveles estructurales principales: el substrato plegado y el neoautóctono $(7,8)$.

El substrato plegado de Cuba está constituido por distintos tipos de terrenos de naturaleza continental y oceánica cuyo origen y evolución tuvieron lugar fuera de los límites del territorio cubano actual. Entre las formaciones de naturaleza continental se encuentran varias estructuras formadas por rocas sedimentarias, como las calizas, dolomitas, silicitas, areniscas y algunas evaporitas (7).

Entre los sedimentos de naturaleza oceánica se encuentran la asociación ofiolítica, conformada por rocas principalmente de origen volcánico, que forma parte de un cinturón longitudinal en la región septentrional de la isla de Cuba; el arco volcánico del Cretácico, constituido por un antiguo archipiélago de islas volcánicas con límites bien definidos; y el arco volcánico del Paleógeno, que surgió en una de las etapas más dinámicas de la evolución geológica de Cuba, la etapa CretácicaPaleogénica $(8,9)$.

En estos sedimentos de naturaleza oceánica predominan básicamente las rocas volcánico-sedimentarias y las intrusivas. En el primer grupo se destaca una gran variedad de lavas, tobas, tefroides, silicitas y tufitas, con frecuencia intercaladas con rocas calizas, arenisca, margas y otras propiamente sedimentarias. En el segundo grupo aparecen con mayor frecuencia gabros, dioritas, granodioritas y cuarzodioritas y, en menor cuantía, granitos y sienitas, además de pegmatitas, pórfidas y aplitas (8).

Las rocas del neoautóctono se formaron después de la consolidación del substrato plegado, o sea, a partir del Eoceno Superior tardío, y se desarrollaron básicamente en el mismo lugar que hoy ocupa el territorio de Cuba. Esta es la razón por la cual se puede considerar la geología del neoautóctono como la verdadera geología de Cuba, formada fundamentalmente por rocas carbonatadas, entre las que se destacan las calizas, dolomitas y margas, entre otras $(7,8)$.

En el presente trabajo se analiza la aparición natural del ión fluoruro en las aguas de consumo y su asociación con algunas variables geológicas y geográficas del territorio cubano, empleando como herramienta de trabajo un sistema de información geográfica.

\section{MATERIALES Y MÉTODOS}

Se estudiaron 753 localidades de 1000 o más habitantes donde se midieron, entre noviembre de 1998 y oc- 
tubre de 1999, las concentraciones de fluoruro en las fuentes de abastecimiento de agua de consumo distribuidas a lo largo de Cuba.

Las variables seleccionadas para el estudio fueron: 1) la concentración de fluoruro en fuentes de abastecimiento de aguas de consumo de poblaciones de 1000 habitantes o más; 2) las características geológicas del territorio según el origen y la evolución de Cuba; 3) los alineamientos (asociaciones de determinados elementos del relieve y el paisaje dispuestos de forma alineada). ${ }^{2}$ 4) los tipos de fuente donde se midieron las muestras; y 5) las categorías del relieve (llanuras y montañas). Estas variables se seleccionaron por ser las de mayor importancia para los objetivos trazados (3, 10, 11-13).

Se empleó como herramienta de trabajo el sistema de información geográfica MapInfo Professional v. 5.5 por ser uno de los sistemas más difundidos mundialmente, así como por su fácil utilización y su eficacia en la integración de información variada (14-16). Para el análisis e interpretación de la información se utilizó el método de superposición con una escala de trabajo de 1:250 000 .

Los datos sobre las concentraciones de fluoruro en aguas de consumo de poblaciones de 1000 habitantes o más se obtuvieron del proyecto Evaluación del Impacto del Programa de la Fluoración de la Sal de Consumo en Cuba, llevado a cabo por la Dirección Nacional de Estomatología del Ministerio de Salud Pública y del Instituto Nacional de Higiene, Epidemiología y Microbiología en el período comprendido entre noviembre de 1998 y octubre de 1999, en el que se obtuvieron muestras de 4588 fuentes de abastecimiento de agua (17).

Las muestras se tomaron directamente en pozos y acueductos. En las localidades que no disponían de acueductos se recolectaron muestras de agua de consumo en 49 viviendas to-

\footnotetext{
2 Los elementos que con más frecuencia dan lugar a alineamientos son las fallas, zonas de elevado agrietamiento, cadenas de manantiales, colinas, escarpes o escalones del relieve, límites entre formaciones litológicas, entre diferentes tipos de suelo, etc.
}

mando como base el procedimiento para garantizar la calidad de los lotes, con un valor de $\propto=0,05$ y de $d$ (muestras con valores de fluoruro $\geq$ a 0,7 $\mathrm{mg} / \mathrm{L}$ ) inferior a 1.

Las muestras fueron analizadas en el Instituto Nacional de Higiene, Epidemiología y Microbiología por el método potenciométrico con electrodo selectivo de fluoruro, con un equipo portátil Orion modelo 290-A (Orion Research Incorporated, Boston, EUA). El método de ensayo se validó y evaluó en el Instituto Nacional de Higiene, Epidemiología y Microbiología (18), según lo establecido en Standard Methods for the Examination of Water and Wastewater, de la American Public Health Association (APHA), la American Water Works Association (AWWA) y Water Pollution Control (WEF).

Un municipio está constituido por varias localidades o puntos poblacionales, en dependencia de su extensión territorial y de su clasificación como urbano o rural. Cada localidad se ve servida por varias fuentes de abastecimiento de agua, según el número de personas abastecidas, la capacidad de las fuentes y la infraestructura de la red de distribución. Como el mapa digital de puntos poblados de Cuba a una escala de 1:250 000, realizado por la Agencia de Cartografía Digital (Geocuba) (19), no permitía representar gráficamente todas las fuentes de abastecimiento de agua, en este estudio se seleccionó como indicador de cada localidad la fuente de abastecimiento con el valor más alto de fluoruro.

Asimismo, para la confección del mapa municipal de las concentraciones de fluoruro se analizaron todas las localidades muestreadas y se tomó como indicador la localidad con el valor más elevado de fluoruro en las aguas de consumo. Se utilizó el mapa digital con la división político administrativa (hasta nivel municipal) a escala 1: 250000 (20).

El mapa geológico generalizado se confeccionó tomando como base el mapa digital de las formaciones geológicas (escala de 1:250 000) confeccionado por el Centro de Investigaciones del Petróleo junto con Geocuba (21).
La información referente al mapa de alineamientos se obtuvo del Nuevo atlas nacional de Cuba (9), a escala 1:2 000 000, el cual sirvió de base para digitalizar dicha información. El mapa está formado por fallas y estructuras anulares definidas y supuestas, así como por el alineamiento cubano central. Para analizar los tipos de fuente donde se midieron las concentraciones de fluoruro se empleó la base de datos de las concentraciones de fluoruro en aguas de consumo por localidades de 1000 habitantes o más (17).

El análisis del relieve y su relación con la distribución espacial del fluoruro en las aguas de consumo se realizó mediante el mapa digital del relieve a escala 1:250 000 de Geocuba (22).

\section{RESULTADOS}

En $83,1 \%$ de las localidades, las muestras de agua fueron recolectadas en pozos y manantiales (aguas subterráneas), y en el 16,9\% restante se recolectaron en presas y ríos. Todas las localidades con concentraciones altas estuvieron asociadas con pozos (cuadro 1).

Las concentraciones de fluoruro de 0,7 mg/L o superiores se localizan fundamentalmente en las regiones oriental y central de Cuba, destacándose las provincias de Las Tunas, Holguín, Camagüey, Granma, Santiago de Cuba y Villa Clara (figura 1). De las 753 localidades estudiadas, 675 (89,6\%) presentan concentraciones bajas o medias (< 0,7 mg/L). La provincia de Las Tunas se destaca como la más afectada por valores elevados de fluoruro en las aguas.

En el mapa de la distribución municipal de concentraciones de fluoruro se observa que la región oriental de Cuba tiene el mayor número de municipios con al menos una localidad con concentraciones superiores a $0,7 \mathrm{mg} / \mathrm{L}$. A esta región le sigue la central. El occidente del país es el que presenta las menores concentraciones de fluoruro en las aguas de consumo (figura 2).

Como bien se plantea en materiales y métodos, los datos sobre las concentraciones de fluoruro en poblaciones de 1000 o más habitantes se obtuvie- 
CUADRO 1. Localidades estudiadas, según el tipo de fuente de abastecimiento y el contenido de fluoruro ( $n=753)$. Cuba, 2001

\begin{tabular}{|c|c|c|c|c|c|c|c|c|c|c|}
\hline \multirow[b]{3}{*}{ Contenido de fluoruro } & \multicolumn{10}{|c|}{ Tipo de fuente de abastecimiento } \\
\hline & \multicolumn{2}{|c|}{ Pozo } & \multicolumn{2}{|c|}{ Manantial } & \multicolumn{2}{|c|}{ Río } & \multicolumn{2}{|c|}{ Represa } & \multicolumn{2}{|c|}{ Total } \\
\hline & No. & $\%$ & No. & $\%$ & No. & $\%$ & No. & $\%$ & No. & $\%$ \\
\hline Medio & 63 & 8,4 & 1 & 0,1 & 1 & 0,1 & 1 & 0,1 & 66 & 8,8 \\
\hline Óptimo & 50 & 6,6 & - & - & 1 & 0,1 & 1 & 0,1 & 52 & 6,9 \\
\hline Alto & 26 & 3,5 & - & - & - & - & - & - & 26 & 3,4 \\
\hline
\end{tabular}

Nota: Las localidades estudiadas se clasificaron según el contenido más alto de fluoruro que tuviesen sus aguas agua de consumo.

FIGURA 1. Mapa de concentraciones de fluoruro en el agua de consumo, por localidad. Cuba, 2001

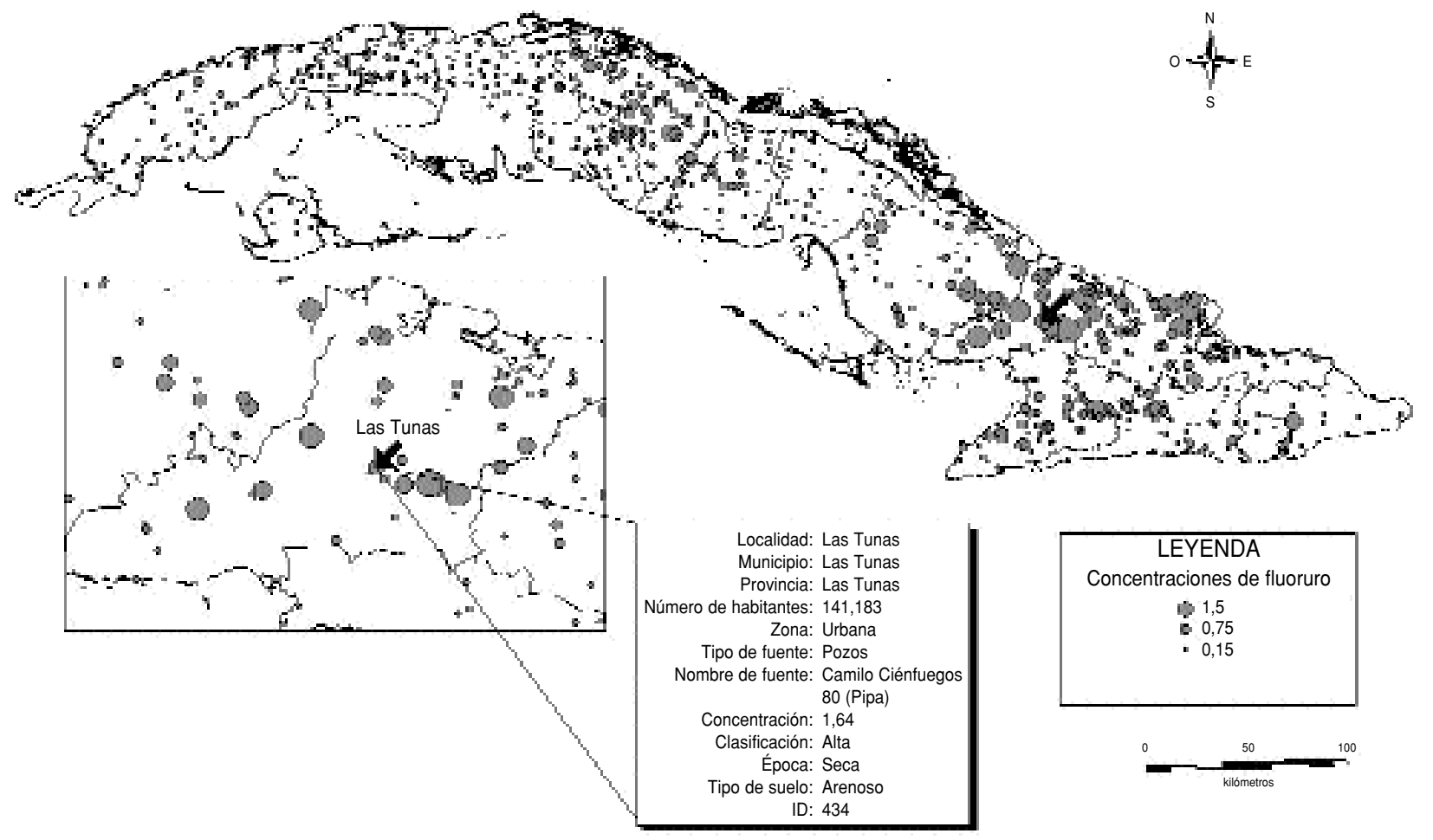

ron del proyecto mencionado. Es importante destacar que los autores de este trabajo también integraron el equipo de investigación de dicho proyecto. De esa base de datos original se derivaron para este estudio otras bases de datos, las cuales permitieron elaborar algunos de los mapas y comparar la información con otras variables.

Las localidades con altas concentraciones de fluoruro natural se encuentran fundamentalmente sobre las rocas volcánico-sedimentarias e intrusivas del arco volcánico del Cretácico, entre las que se destacan las tobas, las andesitas, las lavas y los basaltos, así como granitos, granodioritas, gabros y anfibolitas (figura 3), según reveló la superposición del mapa de concentraciones de fluoruro en aguas de consumo por localidad con el mapa geológico generalizado a escala 1:250 000, en el que aparece la distribución geográfica de las rocas de los arcos volcánicos del Cretácico y del Paleógeno, las ofiolitas septentrionales, así como las rocas del neoautóctono unidas a los sedimentos del substrato plegado de naturaleza continental, pues en ambos casos predominan las rocas sedimentarias y sedimentario-metamorfizadas.

El mapa de alineamientos superpuesto a las localidades donde se determinaron las concentraciones de fluoruro en aguas de consumo y a la geología, se subdividió por regiones (occidental, central y oriental). En la región oriental, la presencia de fluoruro en las aguas de consumo está asociada con los alineamientos de la corteza terrestre, no solo en los complejos rocosos de origen volcánico-sedimen- 


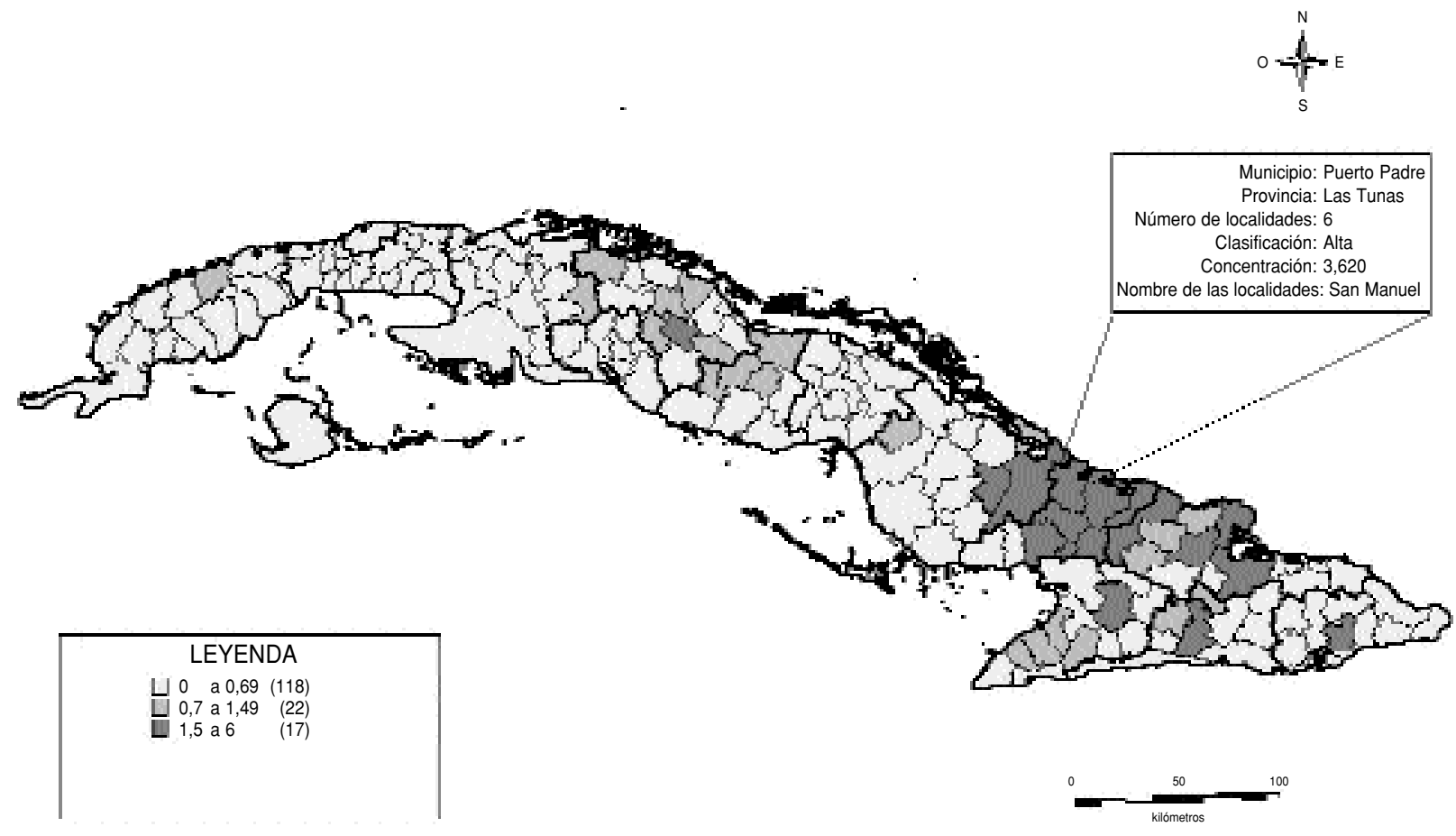

FIGURA 3. Mapa geológico general con las concentraciones de fluoruro en el agua de consumo. Cuba, 2001

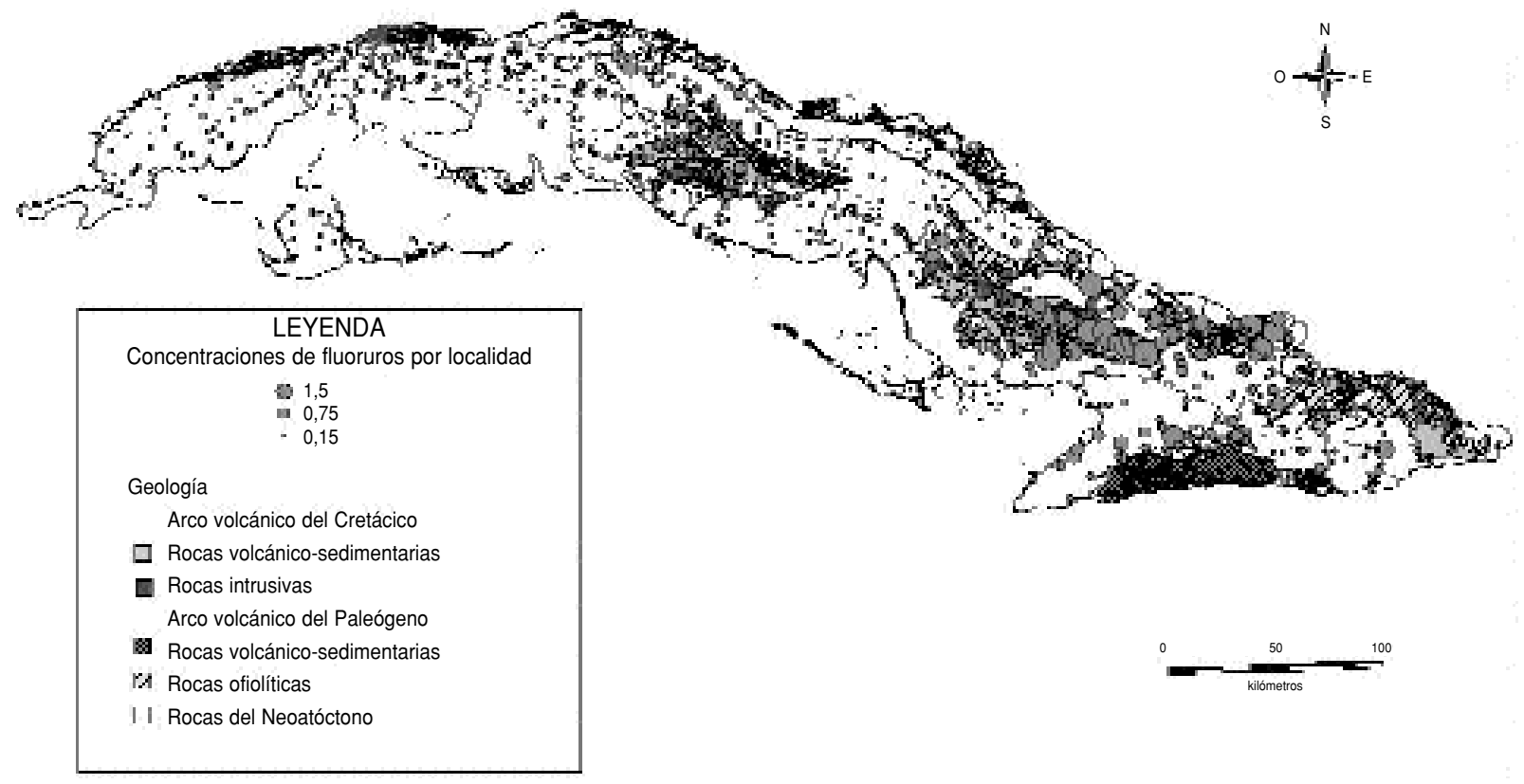

tario e intrusivo, sino también en las rocas carbonatadas (figura 4 ).

El relieve es otra de las variables estudiadas. La región oriental del territo- rio cubano se caracteriza por un predominio de zonas montañosas, donde se encuentran las mayores alturas de Cuba. En esta región, los movimientos neotectónicos verticales del relieve provocan nuevos desniveles y fallas que pueden influir en el escurrimiento superficial y subterráneo de las aguas 
FIGURA 4. Mapa de alineamientos y concentraciones de fluoruro en el agua de consumo. Región central de Cuba, 2001

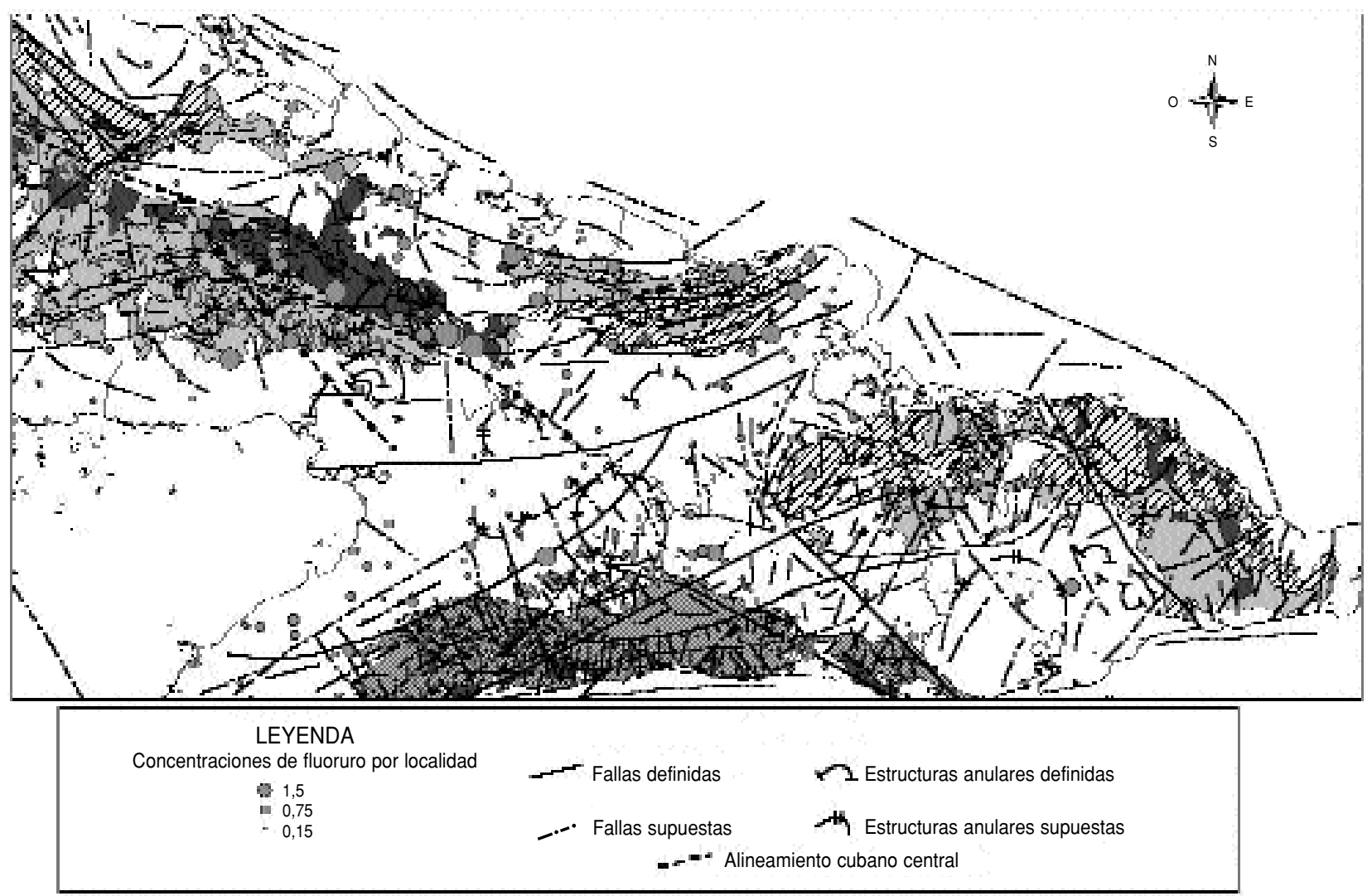

y por tanto en la distribución del fluoruro. Es por ello que en las partes más bajas, próximas a las montañas, se encuentran localidades con concentraciones de fluoruro iguales o superiores a $0,7 \mathrm{mg} / \mathrm{L}$ (figura 5).

\section{DISCUSIÓN}

Al analizar la distribución de las concentraciones de fluoruro en las aguas de consumo en Cuba se observó que la región más afectada es la zona oriental del país, seguida de la zona central. Estos resultados coinciden con los obtenidos en la década de 1970 por Beato y Surí (4), quienes detectaron bajas concentraciones de fluoruro en las aguas cubanas y encontraron solo algunas zonas con concentraciones altas en la región oriental.

La determinación de las concentraciones de fluoruro por municipio permitió identificar territorios donde no se debe suministrar sal fluorurada por presentar al menos una localidad con concentraciones de fluoruro óptimas o altas $(\geq 0,7 \mathrm{mg} / \mathrm{L})$. Esto evita una ingestión excesiva de fluoruro, la cual puede provocar daños a la salud. También se identificaron los municipios con concentraciones de fluoruro por debajo de ese valor, en los que se debe implementar el Programa de Fluoración de la Sal de Consumo para mejorar la salud bucal de la población.

La presencia de fluoruro en las fuentes de abastecimiento de agua está asociada a la disolución de las rocas ígneas $(2,3,6,11,13)$. Aunque el porcentaje de estas rocas en Cuba es bajo, su presencia - particularmente las del arco volcánico del Cretácico- coincide con las zonas donde las concentraciones de fluoruro en el agua son elevadas.

En las investigaciones realizadas en México por Molina, Carrillo y Cardona en la Sierra Madre Occidental y en la zona de Aguascalientes (23-25), así como por Gutiérrez y Sánchez en el sistema acuífero Tesistán-Toluquilla (26), el contenido de fluoruro en el agua se asocia con la presencia de rocas volcánicas, entre ellas tobas, lavas, basaltos y riolitas. Por otro lado, se ha planteado que el fluoruro predomina en gabros, granitos, dioritas, granodioritas, anfibolitas, peridotitas, piroxenitas y sienitas, así como en la pegmatita (13). Los resultados del presente estudio corroboran parcialmente esos hallazgos.

De las características topográficas y geológicas de un territorio depende que los alineamientos se conviertan en zonas de debilidad - lo que facilita el ascenso a la superficie de aguas cargadas de elementos químicos provenientes de los diferentes complejos rocosos por los que transita- o en zonas de recarga del manto freático. Ambos modelos conducen al aumento de la concentración de fluoruro en las fuentes de abastecimiento de agua. Por su parte, 
FIGURA 5. Mapa del relieve y de las localidades donde se estudiaron las concentraciones de fluoruro en el agua de consumo. Región oriental de Cuba, 2001

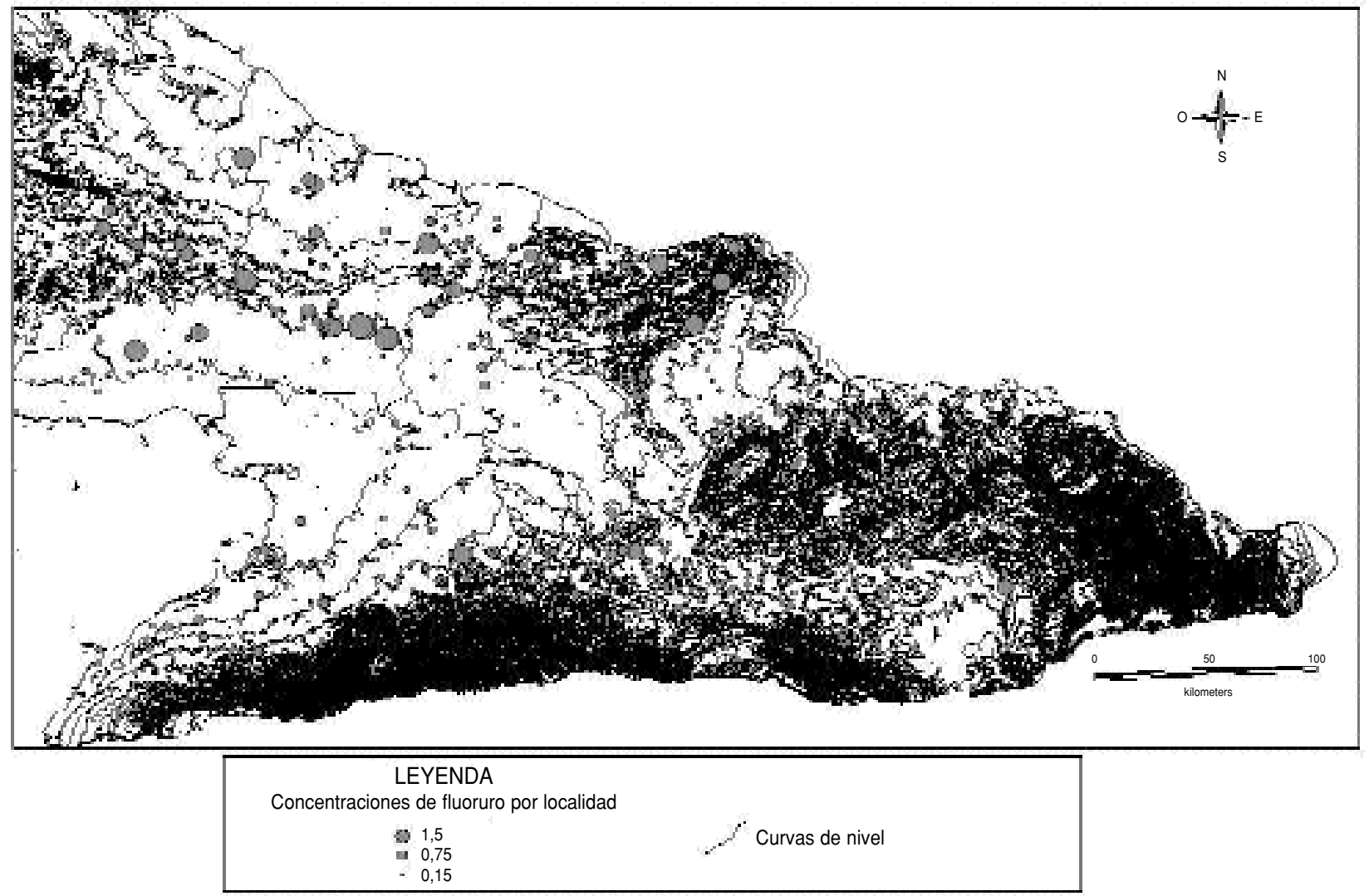

Cederstrom señala que las fallas pueden provocar cambios en la dirección de las aguas subterráneas, cuyo movimiento pueden facilitar o impedir (27).

En algunas localidades que quedan sobre rocas carbonatadas las concentraciones de fluoruro muestran valores óptimos o superiores. Muchas de estas localidades están próximas a los arcos volcánicos del Cretácico y del Paleógeno y a las ofiolitas septentrionales. Estas localidades reciben la influencia de las diferentes fallas y estructuras anulares del territorio, lo que facilita la aparición de fluoruro en lugares donde no se encuentran rocas con un alto contenido de sales de flúor. Los alineamientos son asociaciones de determinados elementos del relieve y el paisaje dispuestos de forma alineada. Los elementos que con más frecuencia dan lugar a ellos son: fallas, zonas de elevado agrietamiento, cadenas de manantiales, colinas, escarpes o escalones del relieve, límites entre formaciones litológicas, entre diferentes tipos de suelo, etc.

Es importante destacar que las concentraciones de fluoruro en las distintas fuentes de abasto de agua donde fueron recolectadas las muestras sufren la influencia de las estaciones lluviosa y seca. La extracción excesiva de aguas subterráneas en algunas zonas reduce el volumen de agua de buena calidad, por lo que la estación seca, en algunas ocasiones, trae consigo una sobreexplotación del manto acuífero y provoca un aumento de la concentración de sales en el agua (26). Se debe señalar que casi todas las muestras fueron recolectadas en pozos y durante la época de sequía. Estas condiciones son las que más favorecen la presencia de concentraciones elevadas de fluoruro en las aguas de consumo.

En Cuba, los sistemas montañosos más importantes están distribuidos con bastante uniformidad, ya que se localizan en el occidente, centro y oriente del país. Según Egorov en su libro Hidrogeología de Cuba, el relieve es una variable geográfica de gran importancia porque determina la dirección del escurrimiento superficial y subterráneo de las aguas (28). Al analizar el mapa del relieve de Cuba y las concentraciones de fluoruro por localidades se puede apreciar que la distribución de los sistemas montañosos que alternan con llanuras facilita la alimentación de las aguas subterráneas. Como resultado, el escurrimiento superficial de las aguas de las zonas más elevadas arrastra diversos compuestos químicos que se depositan en las partes llanas próximas a estos sistemas montañosos. El fenómeno se manifiesta claramente alrededor de las montañas de la Sierra Maestra, donde se encuentran localidades con concentraciones de fluoruro iguales o superiores a $0,7 \mathrm{mg} / \mathrm{L}$. 


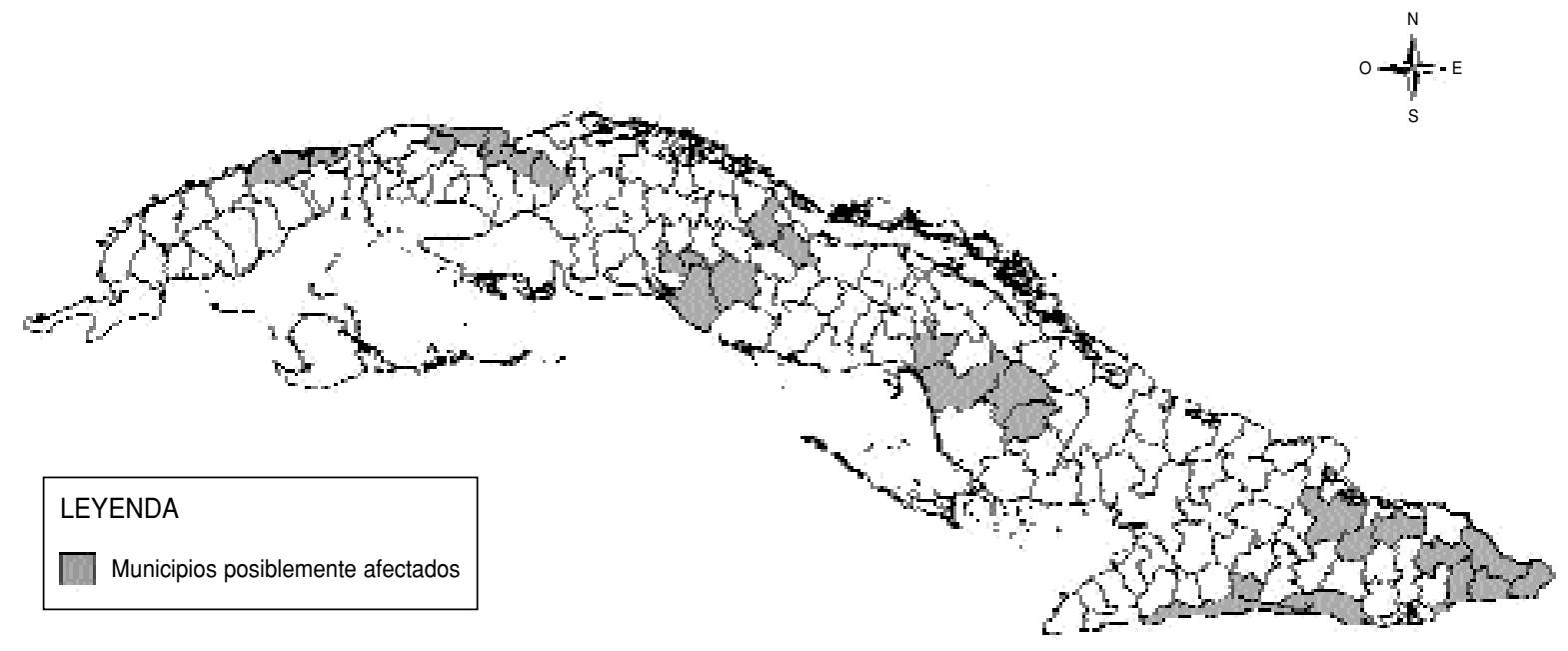

Cabe tener en cuenta que las rocas volcánico-sedimentarias e intrusivas del Cretácico y del Paleógeno, así como las ofiolitas septentrionales, se encuentran fundamentalmente en las zonas más elevadas del territorio oriental cubano, donde predominan las localidades de menos de 1000 habitantes que no fueron estudiadas en el presente trabajo.

Los resultados obtenidos indican que se deben estudiar aquellas zonas de Cuba donde predominan las localidades menores de 1000 habitantes y que por sus características geológicas y geográficas presentan condiciones favorables para la presencia de concentraciones elevadas de fluoruro en las aguas de consumo. Entre los municipios donde deben estudiarse algunas de estas localidades se encuentran los siguientes (figura 6):

- En la región oriental

- Guantánamo: Baracoa, Maisí, Yateras, Imías y San Antonio del Sur
- Santiago de Cuba: Segundo Frente, Tercer Frente, Santiago de Cuba y Guamá

- Holguín: Sagua de Tánamo y Mayarí

- En la región central

- Camagüey: Florida, Camagüey y Jimaguayú

- Ciego de Ávila: Baraguá

- Cienfuegos: Cumanayagua, Cruces y Palmira

- Villa Clara: Manicaragua, Remedios y Camajuaní

- En la región occidental

- Matanzas: Matanzas, Limonar y Jovellanos

- La Habana: Santa Cruz del Norte, Mariel y Jaruco

- Pinar del Río: Bahía Honda.

Los sistema de información geográfica son cada día más utilizados en estudios de salud ambiental y constituyen una herramienta importante para la valoración, comunicación y manejo de datos de riesgo, por lo que pueden facilitar la toma de decisiones encaminadas a proteger la salud de la población.

Mediante el sistema de información geográfica empleado se analizó un conjunto muy variado de información y se logró llegar a una visión integradora de los elementos que influyen en la distribución espacial del fluoruro en las aguas de consumo en Cuba.

En resumen, las concentraciones de fluoruro en las fuentes de agua de consumo de $89,6 \%$ de las poblaciones cubanas con 1000 habitantes o más son bajas o medias, según las normas establecidas por la OMS. Las concentraciones elevadas de fluoruro están asociadas fundamentalmente a las rocas volcánico-sedimentarias e intrusivas del Cretácico. Las características geológicas y geográficas de un territorio pueden ayudar a identificar zonas con concentraciones óptimas y altas de fluoruro en las aguas de consumo.

\section{REFERENCIAS}

1. Organización Panamericana de la Salud. Taller regional de vigilancia epidemiológica y control de calidad de los programas de fluoración de la sal. Quito: Organización Panamericana de la Salud; 1998.

2. González R. Memorias. Primer Curso de Formación de Líderes en Programas de Fluora- ción de la Sal. San José: Programa de Fluoración de la Sal de Costa Rica; 1991.

3. Maier F. Fluoración del agua potable. Washington, D.C.: Organización Panamericana de la Salud; 1971. (Publicación Científica No. 203).

4. Beato O, Surí A. Calidad de las aguas de las principales fuentes de abasto para consumo humano de la República de Cuba. En: Memorias del XXIII Congreso Interamericano de Ingeniería Sanitaria y Ambiental (Tomo 1). La Habana; 1992. Pp. 503-517.

5. Diez P, Bacallao J, Martínez C, Cruz M. Evaluación del programa de fluoración del agua en tres comunidades de la provincia La Ha- 
bana. Rev Cub Hig Epidemiol 1993;31(2):8493.

6. Milovski A, Kónonov O. Mineralogía. Moscú: Mir; 1988.

7. Iturralde-Vinet M. Introducción a la geología de Cuba. En: Furrazola G, Núñez K, eds. Estudios sobre geología de Cuba. La Habana: Instituto de Geología y Paleontología; 1997. Pp. 36-74.

8. Gutiérrez R, Rivero M. Minigeografía de Cuba. La Habana: Científico-Técnica; 1997.

9. Colectivo de Autores. Nuevo atlas nacional de Cuba. La Habana: Instituto de Geodesia y Cartografía, Instituto de Geografía; 1989.

10. Organización Panamericana de la Salud. La salud y el ambiente en el desarrollo sostenible. Washington, D.C.: Organización Panamericana de la Salud; 2000. (Publicación Científica No. 572).

11. Avendaño A. Control de zonas con flúor natural en el agua dentro de un programa de fluoración de la sal. Fluoración al Día 1995; 5:25-34.

12. Salas M, Chavarría P, Dittel F, Huete R, González R. Prevalencia de fluorosis dental en poblaciones de diferente altitud que consumen sal fluorurada. Fluoración al Día 1995;5:2-9.

13. Rankama K, Sahama T. Geoquímica. Madrid: Aguilar, S.A.; 1962.

14. Rodríguez D, Espiñeira J. Sistemas de información geográfica (GIS). PCWorld 1998;197222.
15. Cartwright J. MapInfo offers easy mappig and database management. GIS World 1992;3(2): 78-80.

16. MapInfo Coorporation. MapInfo Professional v. 5.5. Reference Guide. New York: MapInfo Corp; 1999.

17. Sosa M, García M, Cuéllar L, Cangas R, Rodríguez L. Evaluación del Impacto del Programa de la Fluoración de la Sal de Consumo en Cuba. La Habana: MINSAP; 1999.

18. García M, Sosa M, Cuéllar L, Rodríguez L, Canga R. Sistema de vigilancia de fluoruro en aguas de consumo en Cuba. Rev Cub Hig Epidemiol 2002;40(2):136-142.

19. Cuba. Puntos poblados del territorio nacional. [Mapa general]. La Habana: Agencia de Cartografía Digital (Geocuba); 1999.

20. Cuba. División Político-administrativa. [Mapa general]. La Habana: Agencia de Cartografía Digital (Geocuba); 1999.

21. Cuba. Constitución geológica. [Mapa temático]. La Habana: Centro de Investigaciones del Petróleo (CEINPET); 1999.

22. Cuba. Relieve. [Mapa topográfico]. La Habana: Agencia de Cartografía Digital (Geocuba); 1999.

23. Molina-Maldonado A, Cardona A, Carrillo J. Diferenciación hidrogeoquímica de flujo de agua subterránea en la zona de Aguascalientes. En: Memorias del III Congreso Latinoamericano de Hidrología Subterránea. Noviembre de 1996, San Luis Potosí, México.
México D.F.: Guevara Impresores S.A.; 1996. Pp. 79-94.

24. Cardona A, Carrillo J. Mecanismos naturales de control de fluoruros en sistemas regionales de flujo: Sierra Madre Occidental. En: Memorias del III Congreso Latinoamericano de Hidrología Subterránea. Noviembre de 1996, San Luis Potosí, México. México D.F: Guevara Impresores S.A.; 1996. Pp. 67-75.

25. Carrillo J, Cardona A. Relación agua subterránea y ambiente. En: Memoria del Simposio Internacional de Aguas Subterráneas. Diciembre 7-9 de 1998, León, Guanajuato, México. León: Sociedad Mexicana de la Ciencia del Suelo; 1998. Pp. 123-136.

26. Gutiérrez O, Sánchez L. Hidrogeoquímica del sistema acuífero Tesitán-Toluquilla, JaliscoMéxico. En: Memoria del Simposio Internacional de Aguas Subterráneas. Diciembre 7-9 de 1998, León, Guanajuato, México. León: Sociedad Mexicana de la Ciencia del Suelo; 1998. Pp. 19-32.

27. Cederstrom DJ. Aguas subterráneas. Una introducción. 2. ${ }^{\text {a }}$ ed. La Habana: Pueblo y Educación; 1983.

28. Egorov S, Luege J. Hidrogeología de Cuba. La Habana: Instituto Nacional de Recursos $\mathrm{Hi}-$ dráulicos, Instituto Cubano de Recursos Minerales; 1967.

Manuscrito recibido el 24 de octubre de 2002. Aceptado para publicación, tras revisión, el 13 de mayo de 2003.

ABSTRACT Objective. To determine the association between different concentrations of the fluoride ion in drinking water and some geological and geographical variables in Cuba, by using a geographic information system.

Fluoride in drinking water in Cuba and its association with geological and geographical variables
Methods. From November 1998 to October 1999 we studied the fluoride concentration in the sources of drinking water for 753 Cuban localities that had at least 1000 inhabitants. For the information analysis we utilized the MapInfo Professional version 5.5 geographic information system, using the overlaying method. The study variables were the concentration of the fluoride ion in the water sources, the geological characteristics of the area, the alignments (geological characteristics that were found together), the types of water sources, and whether an area was a plain or mountainous. The results were grouped by locality and municipality.

Results. In $83.1 \%$ of the localities, the water samples were collected from wells and springs, and the remaining $16.9 \%$ came from dams and rivers. Of the 753 localities studied, 675 of them $(89.6 \%)$ had low or medium fluoride concentrations (under $0.7 \mathrm{mg} / \mathrm{L}$ ). The eastern region of the country was the one most affected by high fluoride concentrations in the waters, followed by the central region of the country. The majority of the localities with high natural fluoride concentrations were in areas located on Cretaceous volcanic arc rocks. The presence of fluoride in the drinking waters was related to the alignments with the earth's crust, in rock complexes of volcanic-sedimentary origin and of intrusive origin and also in carbonate rocks. However, the highest fluoride concentrations generally coincided with rock complexes of volcanic-sedimentary origin and of intrusive origin. All the localities with high fluoride concentrations in the water were associated with wells.

Conclusions. The fluoride concentration is low or medium in the drinking water sources for $89.6 \%$ of the Cuban localities with at least 1000 inhabitants. Geological and geographical characteristics can help identify areas with optimal or high concentrations of the fluoride ion in the drinking water. 